Pacific Journal of Mathematics

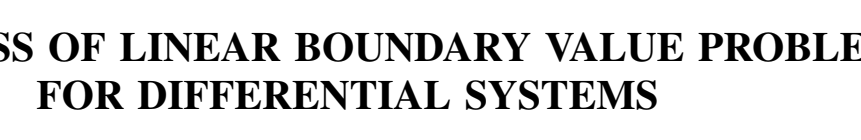




\title{
UNIQUENESS OF LINEAR BOUNDARY VALUE PROBLEMS FOR DIFFERENTIAL SYSTEMS
}

\author{
H. GingOLD
}

\begin{abstract}
A unified approach to "Uniqueness of Boundary Value Problems for Linear and Nonlinear Differential Systems" is expounded. New results are obtained and old one's become transparent.
\end{abstract}

1. Introduction. In this paper we expound a unified approach for determining whether a linear boundary value problem of a non-linear differential system has a unique solution. The advantages of our method are:

(i) It provides a unified approach to linear boundary value problems of a general type.

(ii) It is applicable to linear and non-linear differential systems.

(iii) It provides a tool for investigation of non-linear boundary value problems for non-linear differential systems.

(iv) It makes many old results transparent.

An application of our standpoint to two-point boundary value problems of second order differential systems and equations was demonstrated in Gingold [16].

We will not treat in this paper the uniqueness problem of an $n$-th order differential equation that includes the De la Vallée-Poussin boundary value problem though our method aplies to that problem as well.

We observe from Gingold [16], that many boundary value problems for $n$-th order differential equations exhibit "some sort of singularity." Besides this, the importance of the uniqueness of a De la Vallée-Poussin problem (e.g. see Bessmertnykh [6] for an existence theorem, Levin [34-38] for uniqueness) justifies a special discussion. This will be carried out in Gingold and Gustafson [17].

In $\$ 2$ we introduce assumptions and notations.

In $\$ 3$ we write a necessary and sufficient condition for the boundary value problems of linear differential systems to possess a unique solution. We also point out how the domain of uniqueness of the boundary value problem may be extended.

In $\S 4$ we modify the results of $\S 3$ to nonlinear differential systems.

In $\$ 5$ we illuminate Kim's result [28], and show how nonuniqueness on the boundary $\partial D$ of a simply connected domain $D$, is related to uniqueness of the boundary value problem in $D$.

In $\S 6$ we discuss sufficient conditions for the uniqueness of a special 
form of boundary conditions for "triangular" nonlinear systems. In $§ 7$ we make use of quadratic forms to obtain other sufficient conditions for uniqueness of special boundary value problems.

In $\$ 8$ we characterize "best possible constants" for uniqueness of boundary value problems, and in $\$ 9$ we estimate them.

In $\$ 10$ we conclude with some historical remarks.

\section{Preliminaries. Let}

$$
\vec{y}^{\prime}=\vec{f}(t, \vec{y})
$$

be a nonlinear differential system where $\vec{y}(t)$ is an $n$-column vector

$$
\vec{y}(t)=\left[\begin{array}{c}
y_{1}(t) \\
\vdots \\
\vdots \\
y_{n}(t)
\end{array}\right],
$$

and $\vec{f}(t, \vec{y})$ an $n$ column vector function defined in a domain to be introduced below.

Notation 2.1. Let $D$ be either the interval $[a, b]$, or an open simply connected domain in $\mathbf{C}$ with boundary $\partial D$ which is a piecewisesmooth closed Jordan path. We also denote by $\bar{D}$ the closure of $D$.

Assumption 2.1. The vector function $\vec{f}(t, \vec{y})$ is analytic in $D \times \mathbf{C}^{n}$, for $D \subset \mathbf{C}$.

Assumption 2.2. The vector function $\vec{f}(t, \vec{y})$ is continuous in $(D \cup \partial D) \times \mathbf{C}^{n}$, for $D \subset \mathbf{C}$.

Assumption 2.3. The vector function $\vec{f}(t, \vec{y})$ is a mapping from $[a, b] \times \mathbf{R}^{n}$ into $\mathbf{R}^{n}$ or from $[a, b] \times \mathbf{C}^{n}$ into $\mathbf{C}^{n}$, such that;

(i) for every fixed $\vec{y}, \vec{f}(t, \vec{y})$ is piecewise continuous in $[a, b]$.

(ii) for every fixed $t, \vec{f}(t, \vec{y})$ and its Jacobian, $\vec{f}_{y}(t, \vec{y})$ are continuous in $\mathbf{R}^{n}$ or $\mathbf{C}^{n}$.

Notation 2.2. B.V.P. will be the abbreviation of Boundary Value Problem.

Notation 2.3. A solution of (2.1) will be an analytic $n$-column vector function $\vec{y}(t)$ on $D$, continuously differentiable in $D \cup \partial D$ which satisfies (2.1) subject to assumptions $2.1,2.2$, or a piecewise smooth 
$n$-column vector function $\vec{y}(t)$ on $[a, b]$ which satisfies (2.1) subject to assumption 2.3.

Notation 2.4. Let $\vec{y}(t), \hat{y}(t)$ be two solutions of (2.1), we denote by $B(t, \vec{y}, \vec{y})$ the following matrix

$$
B(t, \vec{y}, \hat{\vec{y}})=\int_{0}^{1} \vec{f}_{y}(t,(\lambda \vec{y}(t)+(1-\lambda) \hat{\vec{y}}(t)) d \lambda
$$

Notation 2.5. The nonhomogeneous linear system (2.3) will be

$$
\vec{y}^{\prime}=f(t, \vec{y})=A(t) \vec{y}+\vec{g}(t)
$$

where $A(t)$ is an $n \times n$ matrix and $\vec{g}(t)$ is an $n$-column vector.

Notation 2.6. The homogeneous linear system (2.2) will be

$$
\vec{y}^{\prime}=\vec{f}(t, \vec{y})=A(t) \vec{y}
$$

where $A(t)$ is an $n \times n$ matrix.

Notation 2.7. We denote by $E$ the $n \times n$ identity matrix.

Notation 2.8. Whenever $\int_{t_{1}}^{t_{2}}$ encountered, for $t_{1}, t_{2}$ two points belonging to a domain $D$, then; if $D=[a, b]$ the integration path is taken along a real segment.

If $D$ is a domain in the complex plane, $\int_{t_{1}}^{t_{2}}$ will be assumed to be taken along a simple Jordan arc imbedded in $D$ and which connects $t_{1}$ and $t_{2}$.

In order to bring out that the integration in the complex plane is taken along a specific simple Jordan arc $\Gamma$, we will use the notation $\int_{t_{\mathrm{r}}}^{t_{2}}$.

Notation 2.9. We denote by $C\left(t, t_{0}\right)$ the solution of the matrix integral equation

$$
C\left(t, t_{0}\right)=E+\int_{t_{0}}^{t} B(s, \vec{y}, \hat{y}) C\left(s, t_{0}\right) d s
$$

where $t, t_{0}$ belong to $[a, b]$ or to $D \cup \partial D$.

Similarly we denote by $Q\left(t, t_{0}\right)$ the solution of the integral equation 


$$
Q\left(t, t_{0}\right)=E+\int_{t_{0}}^{t} A(s) Q\left(s, t_{0}\right) d s .
$$

We recall that $C\left(t, t_{0}\right), Q\left(t, t_{0}\right)$ are given by the infinite series

$C\left(t, t_{0}\right)=E+\int_{t_{0}}^{t} B(s, \vec{y}, \hat{y}) d s+\int_{t_{0}}^{t} B(s, \vec{y}, \hat{y}) \int_{t_{0}}^{s} B\left(s_{1}, \vec{y}, \hat{y}\right) d s_{1} d s$

$$
+\int_{t_{0}}^{t} B(s, \hat{\vec{y}}, \hat{\vec{y}}) \int_{t_{0}}^{s} B\left(s_{1}, \vec{y}, \hat{\vec{y}}\right) \int_{t_{0}}^{s_{1}} B\left(s_{2}, \vec{y}, \hat{\vec{y}}\right) d s_{2} d s_{1} d s+\cdots
$$

$$
Q\left(t, t_{0}\right)=E+\int_{t_{0}}^{t} A(s) d s+\int_{t_{0}}^{t} A(s) \int_{t_{0}}^{s} A\left(s_{1}\right) d s_{1} d s
$$

$$
+\int_{t_{0}}^{t} A(s) \int_{t_{0}}^{s} A\left(s_{1}\right) \int_{t_{0}}^{s_{1}} A\left(s_{2}\right) d s_{2} d s_{1} d s+\cdots
$$

We define the operator $T^{n}, n=0,1, \cdots$ as follows

$$
\begin{gathered}
T^{0}=E, \quad T^{1}=\int_{t_{0}}^{t} A(s) \dot{d} s, \\
T^{n}=\int_{t_{0}}^{t} A(s) \int_{t_{0}}^{s} A\left(s_{1}\right), \cdots, \int_{t_{0}}^{s_{n-2}} A\left(s_{n-1}\right) d s_{n-1} d s_{n-2}, \cdots, d s_{1} d s, \quad n \geqq 1 .
\end{gathered}
$$

We observe that

$$
Q\left(t, t_{0}\right)=\sum_{0}^{\infty} T^{n}
$$

Notation 2.10. Let $\vec{y}(t)$ be a solution of (2.1).

Let $t_{i} \in D, i=1, \cdots, m$ (or $t_{i} \in D \cup \partial D$ ), be $m$ points, not necessarily distinct. Let $P_{\imath}, i=1, \cdots, m$, be $m n \times n$ matrices. Then the boundary conditions will be given by the equation

$$
\sum_{i=1}^{m} P_{i} \vec{y}\left(t_{i}\right)=\vec{C}
$$

where $\vec{C}$ is a given $n$-column vector belonging to $\mathbf{R}^{n}$ or $\mathbf{C}^{n}$.

Throughout the paper, it will be assumed that $\left(\sum_{i=1}^{m} P_{i}\right)$ is invertible.

Notation 2.11. We denote by $\vec{\eta}$ the initial value taken by the solution $\vec{y}(t)$ at the initial point $t_{0}$. 
3. A uniqueness criteria. We are ready now to formulate a necessary and sufficient condition for linear differential systems to possess a unique solution to B.V.P. (2.1), (2.10).

Proposition 3.1. Given B.V.P. (2.1), (2.10) let Assumptions 2.1, 2.2 or Assumption 2.3 be satisfied then:

(i) The B.V.P. (2.1), (2.10) has a unique solution iff

$$
\begin{gathered}
d\left(t_{0}, t_{1}, t_{2}, \cdots, t_{m}\right) \neq 0, \text { where } \\
d\left(t_{0}, t_{1}, t_{2}, \cdots, t_{m}\right)=\operatorname{det}\left(\sum_{i=1}^{m} P_{i}+\sum_{i=1}^{m} P_{1} \int_{t_{0}}^{t_{i}} A(s) Q\left(s, t_{0}\right) d s\right)
\end{gathered}
$$

(ii) Let $L$ and $R$ be $n \times n$ invertible matrices. Then B.V.P. (2.3), (2.10), has a unique solution iff

$$
\operatorname{det}\left[L\left(\sum_{i=1}^{m} P_{i}\right) R+\sum_{i=1}^{m} L P_{1} \int_{t_{0}}^{t_{i}} A(s) Q\left(s, t_{0}\right) R d s\right] \neq 0
$$

(iii) Choose in (3.3) $L=\left(\sum_{i=1}^{m} P_{i}\right)^{-1}$ and $R=E$.

We denote by

$$
\hat{P}_{i}=\left(\sum_{i=1}^{m} P_{i}\right)^{-1} P_{i}
$$

Then B.V.P. (2.1), (2.10) has a unique solution iff

$$
\operatorname{det}\left[E+\sum_{i=1}^{m} \hat{P}_{i} \int_{t_{0}}^{t_{i}} A(s) Q\left(s, t_{0}\right) d s\right] \neq 0 \text {. }
$$

Proof. With the Notation 2.11 , let $\vec{y}(t)$ and $\hat{y}(t)$ be two solutions to B.V.P. (2.3), (2.10). Then by

$$
\begin{aligned}
& \vec{y}\left(t_{i}\right)=Q\left(t_{i}, t_{0}\right) \vec{\eta}=\vec{\eta}+\left(\int_{t_{0}}^{t_{i}} A(s) Q\left(s, t_{0}\right) d s\right) \vec{\eta}, \\
& \hat{y}\left(t_{i}\right)=Q\left(t_{i}, t_{0}\right) \hat{\eta}=\hat{\eta}+\left(\int_{t_{0}}^{t_{i}} A(s) Q\left(s, t_{0}\right) d s\right) \hat{\eta} .
\end{aligned}
$$

Utilizing (3.6), (3.7) one obtains from (2.10) by subtraction (3.8) $\sum_{i=1}^{m} P_{i}[\vec{y}(t)-\hat{y}(t)]=\left[\sum_{i=1}^{m} P_{i}+\sum_{i=1}^{m} P_{i} \int_{t_{0}}^{t_{i}} A(s) Q\left(s, t_{0}\right) d s\right](\vec{\eta}-\hat{\eta})=\overrightarrow{0}$. 
Formula (3.8) immediately yields the results (i) to (iii) of our proposition. An inspection of formula (3.2) shows that if $A=0$ on $D$ then (3.2) is satisfied. This is a first justification to the assumption that $\left(\sum_{i=1}^{m} P_{\imath}\right)$ is invertible. If we let $t_{t}=t$ for $i=1,2, \cdots, m$, then

$$
\begin{aligned}
\operatorname{det}\left[\sum_{i=1}^{m} P_{i}+\sum_{\imath=1}^{m} P_{i} \int_{t_{0}}^{t_{i}} A(s) Q\left(s, t_{0}\right) d s\right] \\
=\left[\operatorname{det}\left(\sum_{\imath=1}^{m} P_{\imath}\right)\right]\left[\operatorname{det}\left(E+\int_{t_{0}}^{t} A(s) Q\left(s, t_{0}\right) d s\right)\right] .
\end{aligned}
$$

From formula (3.9) we get

$$
\operatorname{det}\left(E+\int_{t_{0}}^{t} A(s) Q\left(s, t_{0}\right) d s\right) \neq 0 \quad \text { for } \quad t \in D
$$

therefore if we would like our uniqueness criteria to include initial value problems then assumption on the invertability of $\left(\sum_{i=1}^{m} P_{i}\right)$ is necessary. It is also easily observed from formula (3.2) that if $A$ is "small" then by (2.6), $Q\left(t_{1}, t_{0}\right)$ is also small. Therefore if $A(t)$ is "small" enough then

$$
\left(\sum_{i=1}^{m} P_{i} \int_{t_{0}}^{t_{i}} A(s) Q\left(s, t_{0}\right) d s\right)
$$

is small enough and this implies that the "perturbation" of the invertible matrix $\left(\sum_{i=1}^{m} P_{i}\right)$ will be still invertible to guarantee a unique solution to B.V.P. (2.1), (2.10). The uniqueness criteria for the Nicoletti problem which appears in the literature under the term nonoscillation is a particular case of our formulation.

Proposition 3.2. Assume the systems (2.3) and (2.4) to satisfy Assumptions 2.1, 2.2, or 2.3.

Then B.V.P. (2.3), (2.10) has a unique solution for every vector $\vec{C}$ and every $m$ points $t_{i} \in D, i=1,2, \cdots, m$ iff the only solution to the B.V.P. $\sum_{i=1}^{m} P_{i} \vec{y}\left(t_{i}\right)=\overrightarrow{0}$ of the system (2.4), for every $m$ points $t_{i} \in D$, is the zero solution.

Proof. The difference of two solutions of (2.3) is a solution of the corresponding homogeneous system.

Proposition 3.3. Let (2.3), (2.4) satisfy Assumptions 2.1, 2.2, or 2.3. 
Denote by $P_{N i}, i=1,2, \cdots, n$ the matrices which have all of their entries zero, except the entry in the $(i, i)$ place is 1 .

$$
P_{N i}=\left[\begin{array}{llllll}
0, & & & \uparrow & & \\
& \ddots & & & 0 \\
& & 0 & & & \\
& & 1 & 0 & & \rightarrow \\
& & & \ddots & \ddots & \\
& & & & & \\
& & & & & 0
\end{array}\right] i
$$

Then (2.4) is nonoscillatory on $D$ iff for every $n$ points $t_{i} \in D, i=$ $1,2, \cdots, n$

$$
\operatorname{det}\left[E+\sum_{i=1}^{n} P_{N i} \int_{t_{0}}^{t_{i}} A(s) Q\left(s, t_{0}\right) d s\right] \neq 0
$$

Proof. This follows from the observation

$$
\sum_{i=1}^{n} P_{N i} \vec{y}\left(t_{i}\right)=\left[\begin{array}{c}
y_{1}\left(t_{1}\right) \\
y_{2}\left(t_{2}\right) \\
\vdots \\
y_{n}\left(t_{n}\right)
\end{array}\right]
$$

and Proposition 3.1.

We observe from Proposition 3.1 part (ii) that the B.V.P. (2.10), and the B.V.P. with

$$
\sum_{i=1}^{m} L P_{i} \vec{y}\left(t_{i}\right)=\vec{C}
$$

are equivalent from the point of view of uniqueness.

We make another observation.

Proposition 3.4. Assume $2.1,2.2$ or 2.3 to hold in $D$ and in a larger domain $\hat{D}$ with $D \subset \hat{D}$ and $\hat{D} / D \neq \varnothing$ for the system (2.3). Assume B.V.P. (2.3), (2.10) to possess a unique solution in $D$. Then there exists a domain $\tilde{D}, D \subset \tilde{D} \subset \hat{D}$ such that $\tilde{D} / D \neq \varnothing$, and is such that B.V.P. (2.3), (2.10) has a unique solution in $\tilde{D}$. 
Proof. Since $d\left(t_{0}, t_{1}, t_{2}, \cdots, t_{m}\right)$ is a continuous function in all of its variables for $t_{i} \in D$ and for $t_{i} \in \hat{D}, i=0,1, \cdots, n$, it follows by Proposition 3.1 that $d\left(t_{0}, t_{1}, t_{2}, \cdots, t_{m}\right) \neq 0$ in a domain $\tilde{D}$ with the desired properties.

4. Uniqueness and non-linear systems. We point out in this section the modified results of $\S 3$ which carry over to nonlinear systems.

Proposition 4.1. Let $\vec{y}(t), \hat{y}(t)$, be two solutions of the system (2.1) which satisfies Assumptions 2.1, 2.2 or Assumption 2.3.

Then their difference $\vec{z}(t)$,

$$
\vec{z}(t)=\vec{y}(t)-\hat{y}(t)
$$

satisfies the linear differentiat system

$$
\vec{z}^{\prime}=B(t, \vec{y}, \hat{y}) \vec{z}
$$

and the integral equation

$$
\vec{z}(t)=\vec{z}\left(t_{0}\right)+\int_{t_{0}}^{t} B(s, \vec{y}, \hat{y}) \vec{z}(s) d s .
$$

Proof. Since $\vec{y}(t), \hat{y}(t)$ are solutions of (2.1) we obtain by subtraction

$$
\vec{y}^{\prime}(t)-\hat{\vec{y}}^{\prime}(t)=B(t, \vec{y}, \hat{\vec{y}})(\vec{y}(t)-\hat{\vec{y}}(t))
$$

using Notation 2.4. Formula (4.1) yields immediately (4.2) and (4.3).

Proposition 4.2. With the assumptions of Proposition 4.1 we have

(i) The B.V.P. (2.1), (2.10) has a unique solution if

$$
d\left(t_{0}, t_{1}, \cdots, t_{m}, \vec{y}, \hat{y}\right) \neq 0
$$

where

$$
d\left(t_{0}, t_{1}, \cdots, t_{m}, \vec{y}, \hat{y}\right)=\operatorname{det}\left[\sum_{i=1}^{m} P_{i}+\sum_{i=1}^{m} P_{i} \int_{t_{0}}^{t_{t}} B(s, \vec{y}, \hat{y}) C\left(s, t_{0}\right) d s\right]
$$

(ii) Let $L$ and $R$ be $n \times n$ invertible matrices, then B.V.P. (2.1), (2.10) has a unique solution if 


$$
\operatorname{det}\left[L\left(\sum_{i=1}^{m} P_{i}\right) R+\sum_{i=1}^{m} L P_{i} \int_{t_{0}}^{t_{i}} B(s, \vec{y}, \hat{y}) C\left(s, t_{0}\right) R d s\right] \neq 0
$$

(iii) Choose in (4.7) $L=\left(\sum_{i=1}^{m} P_{i}\right)^{-1}$ and $R=E$ using the notation of (3.4), we have that B.V.P. (2.1), (2.10) has a unique solution if

$$
\operatorname{det}\left[E+\sum_{i=1}^{m} \hat{P}_{i} \int_{t_{0}}^{t_{1}} B(s, \vec{y}, \hat{y}) C\left(s, t_{0}\right) d s\right] \neq 0 \text {. }
$$

Proof. By use of Proposition 4.1 we deduce that if $\vec{y}(t), \hat{y}(t)$, are two solutions which satisfy (2.1), (2.10), then their difference satisfies the linear differential system (4.2). We deduce further that

$$
\left(\sum_{i=1}^{m} P_{i}+\sum_{i=1}^{m} P_{i} \int_{t_{0}}^{t_{i}} B(s, \vec{y}, \hat{y}) C\left(s, t_{0}\right) d s\right)(\vec{\eta}-\hat{\eta})=\overrightarrow{0}
$$

Formula (4.9) implies that if (4.5) holds then $\vec{\eta}-\hat{\eta}=\overrightarrow{0}$. Since our system is locally Lipschitzian, $\vec{\eta}=\hat{\eta}$ implies $\vec{y}(t)=\hat{y}(t)$ for every $t \in D$ and parts (i) to (iii) follow.

We turn now to an analog of Proposition 3.4.

Proposition 4.3. Let Assumptions 2.1,2.2 or 2.3 hold on a domain $\bar{D}$ and $\hat{D}$.

Let $\bar{D} \subset \hat{D}$ and $\hat{D} / D \neq \varnothing$.

Let $d\left(t_{0}, t_{1}, \cdots, t_{m}, \vec{y}, \hat{y}\right) \neq 0$ for any two solutions of (2.1), for all $t_{i} \in \bar{D}, i=0,1, \cdots, m$, (including $\vec{y}(t)=\hat{y}(t)$ ).

Assume B.V.P. (2.1), (2.10) to possess a unique solution in $\bar{D}$ for $t_{i} \in \bar{D}, i=D, 1, \cdots, m$.

Assume that from the family of pairs of distinct solutions of B.V P. (2.1), (2.10) in $\hat{\bar{D}}$ it is always possible to choose a uniformly convergent subsequence of pairs of solutions. Then B.V.P. (2.1), (2.10) has a unique solution for all $t_{i} \in \tilde{D}, i=0,1, \cdots, m$ where $\tilde{D}$ is a domain such that $\bar{D} \subset \tilde{D} \subset \bar{D}$ and $\tilde{D} / \bar{D} \neq \varnothing$.

Proof. Assume on the contrary that there does not exist a $\tilde{D}$ with the required properties.

Let $D_{r}$ be a sequence of connected domains such that $\bigcap_{r=1}^{\infty} D_{r}=\bar{D}$, $\bar{D} \subset, \cdots, \subset D_{r}, \cdots, \subset D_{3} \subset D_{2} \subset D_{1}$ and $D_{r} / \bar{D} \neq \varnothing$. Then there exist $m$ tuples $\left(t_{1 r}, \cdots, t_{m r}\right), r=1,2, \cdots$, with at least one coordinate in $D_{r} / \bar{D}$, and there exist pairs of distinct solutions $\left\langle\vec{y}_{r}(t), \hat{\vec{y}}_{r}(t)\right\rangle$ in $\bar{D}_{r}$ such that

$$
d\left(t_{1 r}, \cdots, t_{m r}, \vec{y}_{r}, \hat{y}_{r}\right)=0
$$


and such that

$$
\lim _{r \rightarrow \infty} \vec{y}_{r}(t)=\vec{y}(t) \text { and } \lim _{r \rightarrow \infty} \hat{y}_{r}(t)=\hat{y}(t) .
$$

Choose now in (4.10) a subsequence of $r=1,2, \cdots$ namely $r_{k} \rightarrow \infty$ with $k \rightarrow \infty$ such that $t_{i r k} \rightarrow t_{i}$ and $t_{i} \in \bar{D}$. This yields a contradiction to our assumption.

\section{Nonuniqueness.}

Proposition 5.1. Let Assumptions 2.1, 2.2 hold for the system (2.3).

Assume B.V.P. (2.3), (2.10) has a unique solution for all $t_{i} \in D$, $i=1, \cdots, m$, and all $\vec{C} \in \mathbf{C}^{n}$, but does not have a unique solution for all $t_{i} \in D \cup \partial D, i=1, \cdots, m$, and all $\vec{C} \in \mathbf{C}^{n}$.

Then there exist two distinct solutions to B.V.P. (2.3), (2.10), with $t_{i} \in \partial D, i=1, \cdots, m$.

Proof. This theorem is a corollary of the maximum principle. Consider $d\left(t_{0}, t_{1}, t_{2}, \cdots, t_{m}\right)$ as a function of $m$ variables in the closed domain $(D \cup \partial D)^{m}$ and also as a function of $m$ variables in the open domain $D^{m}$.

Consider now a sequence of simply connected open domains $D_{r}$,

$$
\begin{gathered}
r=1,2, \cdots \\
D_{1} \subset D_{2} \subset D_{3}, \cdots, \subset D_{r} \subset D .
\end{gathered}
$$

Denote their boundaries by $\partial D_{r}$ and let $\partial D_{i} \cap \partial D_{j}=\varnothing$ for $i \neq j$ and $\partial D_{r} \cap \partial D=\varnothing$. Let also $\bigcup_{r=1}^{\infty} D_{r}=D$.

By Proposition 3.1, $d\left(t_{0}, t_{1}, \cdots, t_{m}\right) \neq 0$ for $t_{i} \in D_{r}, \quad r=1,2, \cdots$. Therefore by the maximum principle

$$
\min \left|d\left(t_{0}, t_{1}, \cdots, t_{m}\right)\right|=\left|d\left(t_{0}, t_{1 r}, t_{2 r}, \cdots, t_{m r}\right)\right| \quad t_{i} \in D_{r} \cup \partial D_{r}
$$

where $t_{r r} \in \partial D_{r}, i=1,2, \cdots, m$.

This follows from the fact that our function $d\left(t_{0}, t_{1}, \cdots, t_{m}\right)$ is analytic in $\left(D_{r} \cup \partial D_{r}\right)^{m}$.

Let $\left(t_{1}, \cdots, t_{m}\right)$ be an $m$-tuple in $(D \cup \partial D)^{m}$ such that $d\left(t_{0}, t_{1}, \cdots, t_{m}\right)=0$ and such that at least one of the coordinates $t_{i}$ belongs to $\partial D$.

Choose a sequence $\left(t_{1 r}, \cdots, t_{m r}\right)$ of $m$-tuples which converges to $\left(t_{1}, \cdots, t_{m}\right)$ for $r \rightarrow+\infty$, such that $t_{i r} \in D_{r} \cup \partial D_{r}$.

By (5.1) we have 


$$
\left|d\left(t_{1 r}, \cdots, t_{m r}\right)\right| \geqq\left|d\left(\hat{t}_{1 r}, \cdots, \hat{t}_{m r}\right)\right|, \quad t_{i r} \in D_{r} \cup \partial D_{r}, \quad \hat{t}_{i r} \in \partial D_{r} .
$$

Choose a subsequence from $r=1,2, \cdots$ such that $\left(\hat{t}_{1 r_{k}}, \cdots, \hat{t}_{m r_{k}}\right)$ tends to some point $\left(\hat{t}_{1}, \cdots, \hat{t}_{m}\right)$ for $k \rightarrow \infty, \hat{t}_{i} \in \partial D$.

Since

$$
\lim _{k \rightarrow \infty}\left|d\left(t_{0}, t_{1 r_{k}}, \cdots, t_{m r_{k}}\right)\right|=0
$$

we obtain $d\left(\hat{t}_{1}, \cdots, \hat{t}_{m}\right)=0$ and the result follows.

We thus obtain Kim's result [28].

Proposition 5.2. Let (2.4) satisfy assumptions of Proposition 5.1.

Let (2.4) be oscillatory on $D \cup \partial D$ but non-oscillatory on $D$. Then (2.4) is oscillatory on $\partial D$.

Proof. Choose in Proposition 5.1 the matrices $P_{N_{l}}$ given by (3.11), $m=n$, and observe by Proposition 3.3 the equivalence of nonoscillation and the uniqueness of B.V.P. (2.4), (2.10).

6. A sufficient condition for uniqueness. In this section we show that a special form of the matrices in (2.10) combined with a proper form of the vector function $\vec{f}(t, \vec{y})$ leads to uniqueness of B.V.P. (2.1), (2.10).

Proposition 6.1. Let Assumptions 2.1, 2.2 or 2.3 be satisfied. Let $\vec{f}(t, \vec{y})$ be such that

$$
\vec{f}_{i}(t, \vec{y})=f_{i}\left(t, y_{1}, \cdots, y_{i}\right), \quad i=1,2, \cdots, m,
$$

namely that $\vec{f}_{i}(t, \vec{y})$ depends on $y_{1}, \cdots, y_{1}$ but not on $y_{i}+1, \cdots, y_{n}$.

Let the matrices $P_{i}$ which appear in (2.10) be such that;

$P_{i}$ are lower triangular matrices having all elements of the diagonals of $P_{i}$ zero except the element in the $(i, i)$ place.

In each $P_{i}$ the first $i-1$ rows have zero elements. Also, take $n=m$ in (2.10).

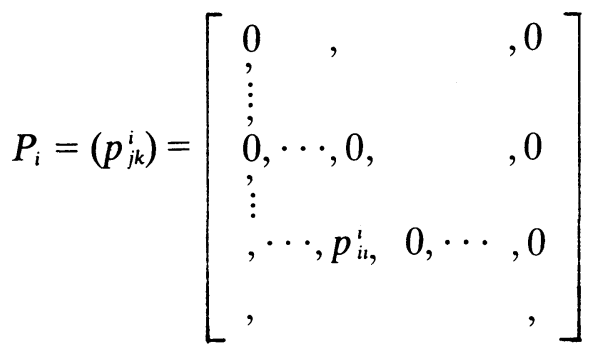

$p_{i i}^{i} \neq 0, p_{j k}^{i}=0$ for $k \geqq j, k \neq j=i$. 
Let $y(t), \hat{y}(t)$ be two solutions of B.V.P. (2.1), (2.10). Then $\vec{y}(t)=\hat{y}(t)$.

Proof. Because of the structure of $\vec{f}(t, \vec{y})$ it is easily verified that $B(s, \vec{y}, \vec{y})$ is a lower triangular matrix and therefore $C\left(s, t_{0}\right)$ is also lower triangular. Inspection of the formula (4.6) reveals that condition (4.5) will be satisfied if the elements on the diagonal of the lower triangular matrix

$$
\left(\sum_{i=1}^{m} P_{i}+\sum_{i=1}^{m} P_{i} \int_{t_{0}}^{t_{i}} B(s, \vec{y}, \hat{y}) C\left(s, t_{0}\right) d s\right), \quad \text { are nonzero. }
$$

But this is true since the elements of the diagonal of the above matrix have the form

$$
p_{i i}^{i} \exp \left(\int_{t_{0}}^{t_{i}} b_{i i}(s, \vec{y}, \hat{y}) d s\right)
$$

where $b_{i i}(s, \vec{y}, \hat{y})$ are the corresponding elements on the diagonal of $B(s, \vec{y}, \hat{y})$.

A more direct proof of this theorem proceeds as follows.

By the structure of the $P_{i}, i=1,2, \cdots, n$ one obtains that $y_{1}(t)$ and $\hat{y}_{1}(t)$ satisfy a first order differential equation and $y_{1}\left(t_{1}\right)=\hat{y}_{1}\left(t_{1}\right)$ which implies $y_{1}(t)=\hat{y}_{1}(t)$ on $D$.

Assume by induction that $y_{j}(t)=\hat{y}_{j}(t)$ on $D$ for $j<n$ and proceed to prove $y_{j+1}(t)=\hat{y}_{j+1}(t)$ on $D$.

This is true since $\hat{y}_{j+1}(t)$ and $y_{j+1}(t)$ are solutions of the same first order differential equation $y^{\prime}=f_{j+1}\left(t, y_{1}(t), \cdots, y_{j}(t), y\right)$ and satisfy the same initial condition at $t_{j+1}$.

7. A positivity criterion for uniqueness. Let us borrow a definition which is a variant of a common concept in the theory of quadratic forms.

Definition 7.1. An $n \times n$ real matrix $A$ will be called positive definite (positive semi-definite) if the quadratic form $x^{t} A x$ is positive definite (positive semi-definite). By this definition $A$ need not be a symmetric matrix.

We denote by $x$, a real $n$ column vector, and by $x^{t}$ its transpose.

Definition 7.2. An $n \times n$ complex matrix $A$ will be called positive definite (positive semi-definite) if for every $n$-column vector $x$, the form $\bar{x}^{\prime} A x$ is "positive definite" ("positive semi-definite"). $\bar{x}$ is the conjugate of $x$. 
Proposition 7.1. Let $A(t)$ be a real $n \times n$ matrix function piecewise-continuous on $[a, b]$. $[a, b]$.

Let $g(t)$ be an $n$-column vector function piecewise-continuous on

Let $A(t), L, R, P_{i}, i=1,2, \cdots, m$ be $n \times n$ real matrices such that;

$L\left(\sum_{i=1}^{m} P_{i}\right) R$ is positive definite,

$L P_{1}\left(\Pi_{j=1}^{r} A\left(t_{j}\right)\right) R$ are positive semi-definite matrices for $i=1,2, \cdots, m$, $n=1,2, \cdots$.

Then B.V.P. (2.3), (2.10) has a unique solution. (We consider only real solutions).

Proof. We use Proposition 3.1, formula (3.2). Multiply the matrix in (3.3) on the right by $x$ and on the left by $x^{t}$.

Then (3.2) holds iff for any nonzero vector $x$ the quadratic form

$$
x^{t} L\left(\sum_{i=1}^{m} P_{i}\right) R x+\sum_{i=1}^{m} x^{t} L P_{i} \int_{t_{0}}^{t_{i}} A(s) Q\left(s, t_{0}\right) R x d s
$$

is positive.

Since by assumption $x^{t} L\left(\sum_{i=1}^{m} P_{i}\right) R x$ is positive it suffices to show that for every $i=1,2, \cdots, m$

$$
V_{i}=x^{t} L P_{i} \int_{t_{0}}^{t_{i}} A(s) Q\left(s, t_{0}\right) R x d s,
$$

is nonnegative. By use of the infinite series (2.9) we get

$$
V_{i}=x^{t} P_{\imath} \int_{t_{0}}^{t_{i}} A(s) \sum_{0}^{\infty} T^{n} R x d s
$$

Our result will follow if $V_{\text {in }}$ given by

$$
V_{\imath n}=x^{t} L P_{\imath} \int_{t_{0}}^{t_{i}} A(s) T^{n} R x d s, \quad i=1, \cdots, m, n=0,1, \cdots,
$$

is nonnegative.

We choose $t_{0}=a$ and proceed to prove that $V_{\text {in }}$ given by

$$
V_{\text {in }}=\int_{t_{0}}^{t_{i}} \int_{t_{0}}^{\zeta} \int_{t_{0}}^{\zeta_{1}}, \cdots, \int_{t_{0}}^{\zeta_{n-1}} x^{t} L P_{i} A(s)\left(\prod_{j=1}^{n} A\left(\zeta_{j}\right)\right) R x d \zeta_{n}, \cdots, d \zeta_{1} d s,
$$

is nonnegative. (The formula (7.5) is valid for $n>1$; its modification for $n=0,1$, is obvious). But this is true since $t_{i} \geqq t_{0}$ and the integrand is a 
nonnegative function, and the result follows. A similar result holds for complex-valued systems.

We state the result as a proposition without proof.

Proposition 7.2. Assume the $n \times n$ matrix function $A(t)$ and the $n$-column vector function $\vec{g}(t)$ to be piecewise-continuous on $[a, b]$.

Let $A(t), L, R, P_{i}, i=1,2, \cdots, m$ be $n \times n$ matrices such that:

$L\left(\sum_{i=1}^{m} P_{i}\right) R$ is a positive definite matrix,

$L P_{i}\left(\sum_{j=1}^{j=r} A\left(t_{j}\right)\right) R$ are positive semi-definite matrices for $i=1,2, \cdots, m$, $r=1,2, \cdots$.

Then B.V.P. (2.3), (2.10) has a unique solution.

We turn to a variant of Proposition 7.1 and 7.2.

Proposition 7.3. Assume the $n \times n$ function matrix $A(t)$ and the $n$ column vector function $\vec{g}(t)$ to be piecewise-continuous on $[a, b]$.

Let $A(t), L, R, P_{i}, i=1,2, \cdots, m$, be $n \times n$ matrices such that;

$L\left(\sum_{i=1}^{m} P_{i}\right) R$ is a positive definite matrix,

$L\left(\sum_{i=1}^{m-\nu} P_{i}\right)\left(\prod_{j=1}^{r} A\left(\zeta_{j}\right)\right) R$, for $\nu=1,2, \cdots, m-1, r=1,2, \cdots$ are posi tive semi-definite matrices.

Then B.V.P. (2.3), (2.10) has a unique solution if $t_{1} \leqq t_{2}, \cdots, \leqq t_{m}$.

Proof. The proof follows by rearranging the terms that appear in the matrix considered in (3.3).

Since

$$
\sum_{i=1}^{m} P_{\imath} \int_{t_{0}}^{t_{i}} A(s) Q\left(s, t_{0}\right) d s=\sum_{\nu=0}^{m-1}\left(\sum_{i=\nu+1}^{m} P_{i}\right) \int_{t_{\nu}}^{t_{\nu+1}} A(s) Q\left(s, t_{0}\right) d s .
$$

We obtain by Proposition 3.1,

$$
\operatorname{det}\left[\sum_{i=1}^{m} P_{i}+\sum_{\nu=0}^{m-1}\left(\sum_{i=\nu+1}^{m} P_{i}\right) \int_{t_{\nu}}^{t_{\nu+1}} A(s) Q\left(s, t_{0}\right) d s\right] \neq 0
$$

implies that B.V.P. (2.3), (2.10) has a unique solution.

We choose in (7.7) $t_{0}=t_{1}$ and repeat the argument of Proposition 7.1 to obtain the desired result.

Our theorem requires that a product of matrices be positive definite. We may expect in some circumstances to use the well known theorems where the signs of minors of a symmetric matrix are used to determine whether it is positive definite. With this in mind, we mention the following lemma.

Lemma 7.1. Let $A$ and $B$ be two $n \times n$ totally positive matrices (See Karlin [25] Ch. 1), then $A B$ is a totally positive matrix. 


\section{Proof. See Karlin [25] Ch. 1, 2.}

We thus have obtained the following proposition.

Proposition 7.4. Let $A(t)$ be an $n \times n$ matrix function and $\vec{g}(t)$ an $n$ vector -function both piecewise continuous on $[a, b]$. Let the matrices $L, R, P_{i}, i=1,2, \cdots, m, \sum_{i=1}^{m} P_{i}, A(t)$ be totally positive.

Let $L\left(\sum_{i=1}^{m} P_{i}\right) R, L P_{\imath}\left(\prod_{j=1}^{\prime=r} A\left(\zeta_{\jmath}\right)\right) R$ be symmetric matrices. Then B.V.P. (2.3), (2.10) has a unique solution.

Proof. By lemma 7.1 all the principal minors of the symmetric matrices that are involved in $V_{1}$ and $V_{\text {in }}$ (7.3), (7.5), are nonnegative. This implies by Gantmacher [15] Ch. X, that $V_{i}$ are nonnegative definite.

Similarly, $L\left(\sum_{i=1}^{m} P_{i}\right) R$ is symmetric and has all its principal minors positive. Thus, all conditions of Proposition 7.1 are fulfilled; and the result follows.

An ExAMPLE. Let $P_{i}, i=1,2, \cdots, m$, be totally positive symmetric matrices which commute with the totally positive symmetric matrix $A$. Let $z(t)$ be a nonnegative, piecewise continuous function on $[a, b]$.

Let the eigenvalues of $\left(\sum_{i=1}^{m} P_{i}\right)$ be positive. Then the B.V.P.

$$
\begin{aligned}
& \vec{y}^{\prime}=a(t) A \vec{y}, \\
& \sum_{i=1}^{m} P_{i} \vec{y}\left(t_{i}\right)=\vec{C} .
\end{aligned}
$$

has a unique solution. This is true by choosing in Proposition 7.4 $L=R=E$.

8. Characterization of best constants. We are going to determine the existence of best constants $\rho$ such that

$$
\int_{a}^{b}\|A(t)\| d t<\rho
$$

will imply that B.V.P. (2.3), (2.10) has a unique solution. First, consider a couple of lemmas borrowed from Schwarz [53].

LEMMA 8.1. With every simply connected domain $D$ which has at least two boundary points, there exists a family of nonintersecting, smooth, closed Jordan arcs $\partial D_{n}$, depending continuously on the parameter $r$, $0<r<1$, such that the interiors of $\partial D_{r}$ are bounded simply-connected domains $D_{r}$. 


$$
\begin{gathered}
D_{r_{1}} \subset D_{r_{2}}, \text { for } r_{1}<r_{2} \\
\partial D_{r_{1}} \cap \partial D_{r_{2}}=\varnothing, \text { for } r_{1} \neq r_{2} \\
\lim _{r \rightarrow 1} \partial D_{r}=\partial D \\
\bigcup_{0<r<1} \partial D_{r}=\bigcup_{0<r<1} D_{r}=D \cup \partial D .
\end{gathered}
$$

Proof. The lemma is a corollary of Riemann's mapping theorem. If $\varphi(z)$ is the univalent function in $|z|<1$ which maps the unit circle onto $D$, then $\partial D_{r}$ are the level curves corresponding to $|z|=r$, and $D_{r}$ are the images of $|z|<r$ under $\varphi(z)$.

LeMma 8.2. Let $A(z)$ be an analytic matrix function in $D$, continuous in $D \cup \partial D$. Define $I(r)$ for $0 \leqq r<1$ to be

$$
I(r)=\int_{\partial D_{r}}\|A(z)\||d z|
$$

and set

$$
I(1)=\lim _{r \rightarrow 1} I(r)
$$

whenever the limit exists.

(If $I(1)<\infty, A(z)$ is said to belong to $H_{1}$ ). Then $\|A(z)\|$ is a continuous subharmonic function in $D$; and $I(r)$ is a nondecreasing function of $r$. Moreover, if $I(1)<\infty$, then there exists a constant $c$ such that

$$
\int_{t_{{ }^{\circ}} \Gamma_{t}}^{t}\|A(s)\||d s| \leqq c \int_{\partial \mathrm{D}}\|A(s)\||d s|=c I(1)
$$

where $t_{0}=\varphi(0), t=\varphi\left(\operatorname{Re}^{i \theta}\right) \in D$, and $\Gamma_{t}$, the path of integration, is the image of the segment $s=r e^{i \theta}, 0 \leqq r \leqq R$.

Proof. This lemma is a restatement of Lemmas 2 and 3 in Schwarz [53].

Definition 8.1. Let \|\| be any norm defined in the set of $n \times k$ matrices, and such that 


$$
\|A B\| \leqq \alpha\|A\|\|B\|
$$

where $A, B$ are $n \times k$ and $k \times \nu$ matrices respectively. The number $\alpha$ may depend on $A$ and $B$ but $\hat{\alpha}$ given by

$$
\hat{\alpha}=\sup \alpha<\infty
$$

is independent of $A$ and $B$. Such a norm will be referred to as an algebra norm.

Proposition 8.1. Let \|\| be an algebra norm.

(i) Let (2.1), (2.3) satisfy Assumptions 2.3.

Then there exists a best possible constant $\rho_{I}, 0<\rho_{I} \leqq \infty$ such that

$$
\int_{a}^{b}\|B(s, \vec{y}, \hat{y})\| d s<\rho_{I}
$$

implies B.V.P. (2.1), (2.10) has a unique solution.

$$
\rho_{I}=\inf \int_{a}^{b}\|A(s)\| d s=\sup r
$$

where the inf is taken over all matrices $A(t)$ piecewise continuous on $[a, b]$ and satisfying

$$
\begin{array}{r}
\operatorname{det}\left(\sum_{i=1}^{m} P_{i}+\sum_{i=1}^{m} P_{i} \int_{t_{0}}^{t_{i}} A(s) Q\left(s, t_{0}\right) d s\right)=0 \\
t_{i} \in D, \quad i=0,1, \cdots, m .
\end{array}
$$

The sup is taken over all $r$ such that

$$
\int_{a}^{b}\|A(s)\| d s<r
$$

implies B.V.P. (2.3), (2.10) has a unique solution.

REMARK. If $A(t)$ is a matrix function on $[a, b]$, and $\vec{y}(t), \hat{y}(t)$, of (8.11), are real valued solutions of (2.1), (2.10), we will obtain some $\rho_{I}$ which will be denoted by $\rho_{I R}$.

If $A(t)$ is a complex valued matrix function on $[a, b]$, and $\vec{y}(t), \vec{y}(t)$, of (8.11) are complex valued vector solutions of (2.1), (2.10), we will obtain some $\rho_{I}$ that will be denoted by $\rho_{I C}$.

Clearly, $\rho_{I C} \leqq \rho_{I R}$. 
(ii) Let (2.1) and (2.3) satisfy Assumption 2.1.

Then there exists a best possible constant $\rho_{D}, 0<\rho_{D} \leqq \infty$ such that for fixed $t_{0} \in D$ and every $t \in D$ connected to $t_{0}$ by a Jordan path $\Gamma \subset D$, the inequality

$$
\int_{t_{0_{\Gamma}}}^{t}\|B(s, \vec{y}, \hat{y})\||d s|<\rho_{D}
$$

implies B.V.P. (2.1), (2.10) has a unique solution.

$$
\rho_{D}=\inf \int_{t_{\Gamma} \Gamma}^{t}\|A(s)\||d s|=\sup r
$$

where the inf is taken over all $A(t)$ which satisfy Assumptions 2.1, and are such that

$$
\operatorname{det}\left(\sum_{i=1}^{m} P_{i}+\sum_{i=1}^{m} P_{i} \int_{t_{0_{\Gamma_{i}}}}^{t_{i}} A(s) Q\left(s, t_{0}\right) d s\right)=0
$$

for $t_{i} \in D, i=0,1, \cdots, m$ and for every set of paths of integration, $\Gamma_{1}$ in $D$, such that (8.17) is true.

The sup is taken over all $r$ such that

$$
\int_{t_{\Gamma}}^{t}\|A(s)\||d s|<r
$$

for $t_{0}$ fixed in $D$, and $t \in D$, (8.18) implies the B.V.P. (2.3), (2.10) has a unique solution. $\Gamma$ is some path of integration imbedded in $D$ which connects $t$ and $t_{0}$.

(iii) Let (2.1) and (2.3) satisfy Assumptions 2.1, 2.2.

Then there exists a best possible constant $\rho_{\partial D}, 0<\rho_{\partial D} \leqq \infty$ such that

$$
\int_{\partial D}\|B(s, \vec{y}, \hat{y})\||d s|<\rho_{\partial D}
$$

implies B.V.P. (2.1), (2.10) possesses a unique solution for $t_{1} \in D \cup \partial D$, $i=0,1, \cdots, m$.

$$
\rho_{\partial D}=\inf \int_{\partial D}\|A(s)\||d s|=\sup r
$$

where the inf is taken over all $A(t)$ such that 


$$
\operatorname{det}\left(\sum_{i=1}^{m} P_{i}+\sum_{i=1}^{m} P_{i} \int_{t_{0}}^{t_{i}} A(s) Q\left(s, t_{0}\right) d s\right)=0
$$

$$
t_{i} \in D, \quad i=0,1, \cdots, m .
$$

The sup is taken over all $r$ such that

$$
\int_{\partial D}\|A(s)\||d s|<r
$$

implies B.V.P. (2.3), (2.10) has a unique solution.

Proof. In view of Proposition 4.1 it is enough to consider linear homogeneous differential systems.

We observe that a best possible constant may be $+\infty$ in the case where $P_{i}=0, i=2,3, \cdots, m$, since then our problem is equivalent to an initial value problem.

From the equation (2.6) one obtains after multiplying it on the left by $A(t)$ and integrating:

$$
\int_{t_{0}}^{t} A(s) Q\left(s, t_{0}\right) d s=\int_{t_{0}}^{t} A(s) d s+\int_{t_{0}}^{t} A(s) \int_{t_{0}}^{s} A(\zeta) Q\left(\zeta, t_{0}\right) d \zeta d s .
$$

By a norm estimation

$$
\begin{aligned}
& \left\|\int_{t_{0}}^{t} A(s) Q\left(s, t_{0}\right) d s\right\| \\
& \quad \leqq \int_{t_{0}}^{t}\|A(s)\||d s|+\int_{t_{0}}^{t} \alpha_{s}\|A(s)\|\left\|\int_{t_{0}}^{s} A(\zeta) Q\left(\zeta, t_{0}\right) d \zeta\right\||d s|
\end{aligned}
$$

By Gronwall's inequality (see Hille [23], Ch. 1)

$$
\left\|\int_{t_{0}}^{t} A(s) Q\left(s, t_{0}\right) d s\right\| \leqq \int_{t_{0}}^{t}\|A(s)\||d s|
$$

$$
+\int_{t_{0}}^{t} \alpha_{s}\|A(s)\|\left(\int_{t_{0}}^{s}\|A(\zeta)\||d \zeta|\right)\left[\exp \left(\int_{s}^{t} \alpha_{\zeta}\|A(\zeta)\||d \zeta|\right)\right]|d s|
$$

Applying integration by parts to the right hand side of (8.25) one obtains

$$
\left\|\int_{t_{0}}^{t} A(s) Q\left(s, t_{0}\right) d s\right\| \leqq \int_{t_{0}}^{t}\|A(s)\|\left[\exp \left(\int_{s}^{t} \alpha_{\zeta}\|A(\zeta)\||d \zeta|\right)\right] d s
$$




$$
\begin{aligned}
& \leqq \int_{t_{0}}^{t}\|A(s)\|\left[\exp \left(\hat{\alpha} \int_{s}^{t}\|A(\zeta)\||d \zeta|\right)\right]|d s| \\
& \leqq \hat{\alpha}^{-1}\left\{-1+\exp \hat{\alpha} \int_{t_{0}}^{t}\|A(s)\||d s|\right\} .
\end{aligned}
$$

Set

$$
\max _{t \in D} \int_{t_{0}}^{t}\|A(s)\||d s|=r
$$

Combining (8.26), formula (3.5), and demanding

$$
\left\|\sum_{i=1}^{m} \hat{P}_{1} \int_{t_{0}}^{t_{i}} A(s) Q\left(s, t_{0}\right) d s\right\|<1
$$

one obtains that

$$
r<\hat{\alpha}^{-1} \ln \left[1+\left(\sum_{l=1}^{m}\left\|\hat{P}_{i}\right\|\right)^{-1}\right]
$$

is sufficient to guarantee (3.5).

(i) The inequality (8.14) guarantees the existence of a $\rho_{I}<0$ such that (8.11) implies uniqueness for B.V.P. (2.1), (2.10). (Choose for instance $t_{0}=a$ ). The proof for the inf sup characterization of the best possible $\rho_{I}$ follows from its definition. Consider the norm \|\|$_{1,2}$ to be that induced on a matrix $A$ by two vector norms \|\|$_{1},\|\|_{2}$ by defining

$$
\|A\|_{1,2}=\sup \frac{\|A \vec{y}\|_{2}}{\|\vec{y}\|_{1}} \text {. }
$$

In the case $m=n, P_{N i}=P_{i},\|\|=\|\|_{1,2}$ defined above, we see that $\rho_{I R}$ coincides with

$$
\inf \int_{a}^{b} \frac{\left\|\vec{y}^{\prime}(t)\right\|_{2}}{\|\vec{y}(t)\|_{1}} d t
$$

where the inf is taken over $\vec{y}(t) \neq \overrightarrow{0}$ and $y_{i}\left(t_{i}\right)=0$.

The result (8.31) is due to Friedland [14]. By his definition of the $\rho_{I}$, it is not clear that $\rho_{I}>0$ and that there exists a best possible $\rho_{I C}$. Our approach yields this information immediately.

(ii) It follows from (8.29) that if for every $t \in D$, there exists a Jordan path $\Gamma \subset D$ such that $\int_{t_{\Gamma}}^{t}\|B(s, \vec{y}, \vec{y})\||d s|<r$ holds, then B.V.P. (2.1), (2.10) has a unique solution. By definition, $\rho_{D}$ is the best possible. 
(ii) By Proposition 5.1 we are guaranteed that B.V.P. (2.3), (2.10) has a unique solution for $t_{i} \in D \cup \partial D$ iff B.V.P. (2.3), (2.10) with $t_{i} \in \partial D_{r}$, $i=0,1, \cdots, m$, for every $r, 0<r \leqq 1$, has a unique solution. Recall Lemmas 8.1, 8.2. By the inequality (8.29) we are guaranteed that there exists $\hat{\rho}_{\partial D}$ such that (8.19) implies B.V.P. (2.1), (2.10) has a unique solution, for $t_{i} \in \partial D, i=0,1, \cdots, m$. By Lemma 8.2 this implies that also

$$
\int_{\partial D_{r}}\|A(s)\||d s|<\hat{\rho}_{\partial D} \quad \text { for } \quad 0<r \leqq 1
$$

which implies that B.V.P. has a unique solution for $t_{i} \in D \cup \partial D, i=$ $0,1, \cdots, m$. This argument shows that $\rho_{\partial D}$ given by $(8.20)$ is the best possible.

Proposition 8.2. With the assumptions of Proposition 8.1, with $m=2$,

$$
2 \rho_{I C} \leqq \rho_{\partial D}
$$

Proof. Because of the Riemann mapping theorem and Lemma 8.1 it is enough to consider the case $D=\{t|| t \mid<1\}$. Let $\exp i \theta_{1}, \exp i \theta_{2}$, $0<\theta_{1} \leqq \theta_{2}<2 \pi$, be two points belonging to $|t|=1$.

Then,

$$
\begin{aligned}
2 \rho_{I C} & >\oint_{|t|=1}\|A(t)\||d t|=\int_{0}^{2 \pi}\|A(\exp i \theta)\| d \theta \\
& =\int_{\theta_{1}}^{\theta_{1}+2 \pi}\left\|A\left(e^{i \theta}\right)\right\| d \theta=2 \int_{\theta_{1}}^{\theta_{0}}\|A(\exp i \theta)\| d \theta \\
& =2 \int_{\theta_{0}}^{\theta_{1}+2 \pi}\|A(\exp i \theta)\| d \theta
\end{aligned}
$$

for some $\theta_{0}, \theta_{1}<\theta_{0}<2 \pi+\theta_{1}$.

By (8.11), B.V.P. (2.10) for the system

$$
\frac{d \vec{y}}{d \theta}=[A(\exp i \theta) i \exp i \theta] \vec{y}
$$

must have a unique solution on each of the intervals $\left[\theta_{1}, \theta_{0}\right],\left[\theta_{0}, \theta_{1}+2 \pi\right]$. (Remark, the best constants $\rho_{I R}$ or $\rho_{I C}$ do not depend on the endpoints $a$, $b$, of an interval.)

Since $\theta_{2}$ must be either in $\left[\theta_{1}, \theta_{0}\right]$ or in $\left[\theta_{0}, \theta_{1}+2 \pi\right]$ our result follows.

The relationship among $\rho_{I}, \rho_{D}, \rho_{\partial D}$ seem to be of a very interesting 
nature. However a general theory linking their magnitudes seems to be lacking.

For the Nicoletti problem there are results indicating that $\rho_{D}=2 \rho_{I R}$ for certain norms.

Compare, for instance, Lasota and Olech [32] and Nehari [45] (who found $\rho_{I R}=\pi / 2$, for the norm induced on $A(t)$ by the Euclidean vector norm) with Schwartz [55] and Nehari [47], who found $\rho_{\partial D}=\pi$.

\section{Estimation of best constants.}

Proposition 9.1. Let assumptions of Proposition 8.1 hold.

(i) If \|\| is an algebra norm, then

$$
\rho_{I R} \geqq \rho_{I C} \geqq 2 \hat{\alpha}^{-1} \ln \left[1+\left(\sum_{l=1}^{m}\left\|P_{i}\right\|\right)^{-1}\right]
$$

$$
\begin{gathered}
\rho_{\partial D} \geqq 2 \hat{\alpha}^{-1} \ln \left[1+\left(\sum_{i=1}^{m}\left\|P_{i}\right\|\right)^{-1}\right] \\
\rho_{D} \geqq \hat{\alpha}^{-1} \ln \left[1+\left(\sum_{i=1}^{m}\left\|P_{i}\right\|\right)^{-1}\right] .
\end{gathered}
$$

(ii) Let $L$ be a matrix in a block partitioned form

$$
L=\left[\begin{array}{c}
L_{1} \\
L_{2} \\
\vdots \\
L_{k}
\end{array}\right], \quad i=1,2, \cdots, k
$$

$$
L=\left(L_{1}, L_{2}, \cdots, L_{k}\right)
$$

then if \|\| is an algebra norm such that

$$
\|L\| \leqq \max _{i}\left\|L_{i}\right\|
$$

$$
\begin{aligned}
\rho_{I R} \geqq \rho_{I C} & \geqq 2 \hat{\alpha}^{-1} \ln \left[1+\left\|\left(P_{1}, \cdots, P_{m}\right)\right\|^{-1}\right] \\
\rho_{\partial D} & \geqq 2 \hat{\alpha}^{-1} \ln \left[1+\left\|\left(P_{1}, \cdots, P_{m}\right)\right\|^{-1}\right] \\
\rho_{D} & \geqq \hat{\alpha}^{-1} \ln \left[1+\left\|\left(P_{1}, \cdots, P_{m}\right)\right\|^{-1}\right] .
\end{aligned}
$$


Proof. (i) We demand that (8.28) will hold, so as to guarantee (3.5). A fortiori, if

$$
\left\|\sum_{i=1}^{m} \hat{P}_{i} \int_{t_{0}}^{t_{i}} A(s) Q\left(s, t_{0}\right) d s\right\| \leqq \sum_{i=1}^{m}\left\|\hat{P}_{i}\right\| \cdot\left\|\int_{t_{0}}^{t_{i}} A(s) Q\left(s, t_{0}\right) d s\right\|
$$

$$
\begin{aligned}
& \leqq\left(\sum_{i=1}^{m}\left\|\hat{P}_{i}\right\|\right) \max _{i}\left\|\int_{t_{0}}^{t_{i}} A(s) Q\left(s, t_{0}\right) d s\right\| \\
& \leqq\left(\sum _ { i = 1 } ^ { m } \| \hat { P } _ { i } \| \left[-1+\exp \hat{\alpha} \int_{t_{0}}^{t_{i}} A(s) \||d s|<1\right.\right.
\end{aligned}
$$

then (8.28) is satisfied.

If $D$ is the interval $[a, b]$, choose $t_{0}$ such that

(9.11) $\frac{1}{2} \int_{a}^{b}\|A(s)\| d s=\int_{t_{0}}^{b}\|A(s)\| d s=\int_{a}^{t_{0}}\|A(s) d s\| \geqq \int_{t_{0}}^{t_{i}}\|A(s)\||d s|$.

Since (8.26) implies

$$
\int_{t_{0}}^{t_{i}}\|A(s)\||d s|<\hat{\alpha}^{-1} \ln \left[1+\sum_{i=1}^{m}\left\|\hat{P}_{i}\right\|\right]
$$

we obtain (9.1) and (9.3).

In order to prove (9.2) we choose on $\partial D$ a point $t_{0}$ such that

$$
\frac{1}{2} \int_{\partial D}\|A(s)\||d s| \geqq \int_{t_{0}}^{t_{1}}\|A(s)\||d s|
$$

and (9.2) follows.

(ii) We proceed as in part (i) and exploit the norm property to obtain

$$
\begin{aligned}
& \left\|\sum_{i=1}^{m} \hat{P}_{i} \int_{t_{0}}^{t_{i}} A(s) Q\left(s, t_{0}\right) d s\right\| \\
& \quad \leqq\left\|\left(\hat{P}_{1}, \hat{P}_{2}, \cdots, \hat{P}_{m}\right)\right\| \max _{i}\left\|\int_{t_{0}}^{t_{i}} A(s) Q\left(s, t_{0}\right) d s\right\| .
\end{aligned}
$$

We use (9.12) and repeat the same arguments as in part (i) to obtain our result.

In particular, choose $m=n$ and $P_{N i}=P_{i}$ given by (3.11). If we also take our norm to be the maximal row norm, then the condition of our proposition part (ii) holds with $\hat{\alpha}=1$. Then $\left\|\left(P_{i}, \cdots, P_{m}\right)\right\|=1$ and we obtain, Schwartz [53], 


$$
\rho_{I R} \geqq \rho_{I C} \geqq 2 \ln 2 \text {. }
$$

It was shown by Friedland [14] that actually $\rho_{I C}=2 \ln 2$.

By Proposition 3.1 it follows that any hypothesis on $A(t)$ which makes the eigenvalues of

$$
\sum_{i=1}^{m} \hat{P}_{i} \int_{t_{0}}^{t_{i}} A(s) Q\left(s, t_{0}\right) d s
$$

lie in the interior of the unit disk serves our purposes. Let us introduce a few notations for the next proposition.

Notation 9.1. Given a matrix $A=\left(a_{i j}\right), i=1, \cdots, n, j=1, \cdots, k$, we denote by $|A|$ the matrix $|A|=\left(\left|a_{i j}\right|\right)$.

Notation 9.2. Given the matrices $A$ and $M$ of the same dimensions, $|A| \leqq M$ means that for every $i$ and $j,\left|a_{i j}\right| \leqq m_{i j}$.

Notation 9.3. We denote by $|\lambda|(A)$ the maximum of the absolute values of the eigenvalues of $A$.

Proposition 9.2. Let the assumptions of Proposition 8.1 hold. Then:

(i) If

$$
|B(t, \vec{y}, \hat{y})| \leqq M \quad \text { for } \quad t \in[a, b]
$$

and

$$
|\lambda|\left(\left(\sum_{i=1}^{m}\left|\hat{P}_{i}\right|\right)\left\{\left[\exp \left(\frac{1}{2}(b-a) M\right]-E\right\}\right)<1\right.
$$

or if

$$
\int_{a}^{b}|B(s, \vec{y}, \hat{\vec{y}})| d s \leqq M
$$

and

$$
\min |\lambda|\left\{(M),\left(\left(\sum_{i=1}^{m}\left|\hat{P}_{i}\right|\right) M(E-M)^{-1}\right)\right\}<1
$$

then B.V.P. (2.1), (2.10) has a unique solution. 
In particular, if $\sum_{i=1}^{m}\left|\hat{P}_{i}\right|=E$, condition (9.18) is replaced by

$$
|\lambda|(M)<2(b-a)^{-1} \ln 2
$$

and condition (9.20) is replaced by

$$
|\lambda|(M)<\frac{1}{2}
$$

(ii) Let $D$ be a simply connected domain in the complex plane such that for any $m$ points in $D, t_{i} \in D$, there are $m$ simple Jordan arcs $\Gamma_{i}$, $i=1, \cdots, m$, connecting those points to a point $t_{0}$ in $D$ such that the length of each Jordan arc does not exceed $l$. Then if

$$
\left|B\left(t_{i}, \vec{y}, \hat{y}\right)\right| \leqq M \quad \text { for } \quad t_{i} \in D
$$

and

$$
|\lambda|\left(\left(\sum_{i=1}^{m}\left|\hat{P}_{i}\right|\right)\{\exp l M-E\}\right)<1
$$

or

$$
\int_{t_{\Gamma_{i}}}^{t_{i}}|B(s, \vec{y}, \hat{y})| d s<M,
$$

and

$$
\min |\lambda|\left\{(M),\left(\left(\sum_{i=1}^{m}\left|\hat{P}_{\imath}\right|\right) M(E-M)^{-1}\right)\right\}<1
$$

then B.V.P. (2.1), (2.10) has a unique solution.

Proof. The proof is based on majorizing the infinite series (2.7). By (2.7) it follows that condition (9.17) implies

$$
\begin{aligned}
\left|\sum_{i=1}^{m} \hat{P}_{i} \int_{t_{0}}^{t_{i}} B(s, \vec{y}, \hat{y}) C\left(s, t_{0}\right) d s\right| & \leqq\left(\sum_{i=1}^{m}\left|\hat{P}_{i}\right|\right)\left(\sum_{k=1}^{\infty} \frac{\left(\frac{1}{2}(b-a) M\right)^{k}}{k !}\right) \\
& =\left(\sum_{i=1}^{m}\left|\hat{P}_{i}\right|\right)\left\{\left[\exp \left(\frac{1}{2}(b-a) M\right)\right]-E\right\}
\end{aligned}
$$

Conditions (9.19) and (9.20) imply by the use of (2.7), that 


$$
\begin{aligned}
\left|\sum_{i=1}^{m} \hat{P}_{\imath} \int_{t_{0}}^{t_{i}} B(s, \vec{y}, \hat{y}) C\left(s, t_{0}\right) d s\right| & \leqq\left(\sum_{i=1}^{m} \hat{P}_{\imath}\right)\left(\sum_{k=1}^{\infty} M^{k}\right) \\
& =\left(\sum_{i=1}^{m} \hat{P}_{i}\right) M(E-M)^{-1}
\end{aligned}
$$

We recall from Gantmacher [15] Vol. 2, Ch. 2, that $|A| \leqq M$ implies

$$
|\lambda|(A) \leqq|\lambda|(M) \text {. }
$$

Since for a function $f$ and an $n \times n$ matrix $A$, we have $\lambda(f(A))=$ $f(\lambda(A))$, it follows that (9.21) and (9.22) hold. A similar discussion leads to the results $(9.24),(9.26)$.

10. Some historical remarks. In 1897 Nicoletti [49], considered the existence and uniqueness of the boundary value problem (2.1), (2.10), with $P_{N i}=P_{i}, m=n$ given by (3.11). His main result may be reformulated as follows:

Given the differential system (2.1) subject to the Assumption 2.3 there exists an interval $[a, c], a<c \leqq b$, such that for every $n$-tuple $\left(t_{1}, t_{2}, \cdots, t_{n}\right), a \leqq t_{i} \leqq c, i=1, \cdots, n$, there exists a unique continuously differentiable solution which satisfies (2.1), (2.10).

Since then, this B.V.P. has been known as the Nicoletti problem. Various generalizations of this B.V.P. have been considered.

For the existence problem, and for the existence and uniqueness for general B.V.P. the reader may consult Antosiewicz [1,2], Conti [8, 10], Grandolfi [19], Lasota and Opial [30,31], Lasota and Olech [32], and their references.

Assuming that a solution to the Nicoletti problem exists, the question of its uniqueness gave rise to a wide literature which uses the key word nonoscillation. If the matrix in (2.4) is the companion matrix of an $n$th order differential equation and the Nicoletti problem has a unique solution for every $t_{i} \in D, i=1, \cdots, n$, and every $\vec{C}$ in (2.10), then the corresponding homogeneous $n$th order linear equation is said to be disfocal in $D$.

For sufficient conditions for a system (2.4) to be nonoscillatory on $[a, b]$, the reader is referred to Lasota and Olech [32], Nehari $[45,46]$, and Friedland [13,14]. For sufficient conditions for $(2.4)$ to be nonoscillatory in a complex domain $D$ when $A(t)$ is analytic in $D$ the reader is referred to Nehari [47], Schwartz [52-55], London and Schwarz [39], and their references. It is possible to distinguish three directions in the investigation of nonoscillation of (2.4).

(I) Formulation of necessary and sufficient conditions for (2.4) to 
be nonoscillatory in $D$. For example, Proposition 3.1 satisfies such a criterion. In general the necessary and sufficient conditions are formulated on derived functions of the coefficient matrix $A(t)$ rather then directly on the matrix $A(t)$. This is many steps before the criteria are applicable and practical.

(II) Sufficient conditions on functionals defined on $A(t)$, to guarantee the nonoscillation of (2.4) for all systems (2.4) were formulated. The main theme which appears in them is as follows.

Let \|\| be an algebra norm. If $\int_{a}^{b} A(s) d s$ is less then $\rho$, where $\rho$ is some positive constant, then (2.4) is nonoscillatory. The subject of finding the appropriate $\rho$ when \|\| is a norm induced on $A(t)$ by vector norms took two courses;

(i) By direct estimation. The main tune in this trend is as follows. If $\vec{y}(t)$ is a nonzero vector solution of (2.4) which satisfies $y_{i}\left(t_{i}\right)=0, i=1, \cdots, n$, then by direct estimation one shows that

$$
\int_{a}^{b} \frac{\left\|\vec{y}^{\prime}(t)\right\|}{\|y(t)\|} d t \geqq \rho
$$

for some $\rho>0$. By using an ad-absurdum argument, $\int_{a}^{b}\|A(s)\| d s \leqq \rho$ is found to guarantee the nonoscillation of (2.4). Lasota and Olech [32], used this geometrical argument to find for \|\|$_{2}$ (the norm induced on $A$ by the vector Euclidean norm) that $\rho=\pi / 2$ is the best constant. This result was reestablished later by Nehari [45]. Ad-absurdum arguments to derive (8.1) as a sufficient condition for nonoscillation in case $D$ is the interval $[a, b]$ or $D$ a domain in the complex plane, were used by Nehari [47], Kim [28], Schwarz [52-55].

(ii) By direct use of calculus of variations for real systems. See Friedland $[13,14]$.

(III) Simplification of the problem. Given a specific norm $\|$, induced on $A$ by a vector norm it may happen that the best constant $\rho$ in (8.1) which guarantees nonoscillation of (2.4) does not depend on the dimension $n$ of the system (2.4). Indeed, this was hinted by Lasota and Olech's work [32], verified by calculus of variations by Friedland [14] for various norms, and established by Nehari [48], for \|\|$_{p}, 1 \leqq p$, where \|\|$_{p}$ are the norms induced on $A$ by the Hölder vector norms. By Friedland [14] it is not true that the best constant $\rho$ is independent of $n$ for every norm.

Another simplification in the search for best constants $\rho$ in (8.1) to imply nonoscillation is due to Nehari [46], who showed that it is enough to consider a "degenerate Nicoletti" B.V.P., namely that $t_{i} \in[a, b]$, 
$i=1, \cdots, n$, may be chosen such that $t_{i}$ coincides either with $a$ or with $b$. (A similar phenomenon occurs in the De la Vallée-Poussin problem whose uniqueness is investigated under the name of disconjugacy of $n$th order linear homogeneous differential equations. See Levin [35]. A third type of simplification for nonoscillation problems in a closed domain $D$ in the complex plane was supplied by Kim [28], who made it possible to substitute the question of nonoscillation of a system (2.4) in $D$, by a question of nonoscillation of the system (2.4) on a closed Jordan arc imbedded in $D$.

(IV) Conditions on the form of the matrix $A(t)$ or on special relations among its elements to imply nonoscillation of (2.4). Not much in this direction seems to exist in the literature.

(V) Necessary conditions on $A(t)$ if $A(t)$ is analytic in $D$ and (2.4) is nonoscillatory. In this direction consult Lavie [33].

Acknowledgement. I wish to thank Professor G. Gustafson whose valuable suggestions helped to bring this paper to its final form.

\section{REFERENCES}

1. H. A. Antosiewicz, Boundary value problems for nonlinear ordinary differential equations, Pacific J. Math., 17 (1966), 191-197.

2. _ Linear problems for nonlinear ordinary differential equations, Proc. U.S.-Japan Sem. Diff. and Funct. Eqns., W. A. Benjamin, New York, 1967, 1-11.

3. P. R. Beesack, Integral inequalities of the Wirtinger type, Duke Math. J., 25 (1958), 477-498.

4. _ - On the Green's function of an N-point boundary value problem, Pacific J. Math., 12 (1962), 801-812.

5. R. Bellman, Introduction to Matrix Analysis, McGraw-Hill, 1970.

6. G. A. Bessmertnykh, Existence and uniqueness of solutions of a multipoint De la Vallée-Poussin boundary-value problem for nonlinear differential equations, Translated from Differential'nye Uravneniya, Vol. 6, No. 2, (1970), 298-310.

7. E. A. Coddington and N. Levinson, Theory of Ordinary Differential Equations, McGraw-Hill, New York, 1955.

8. R. Conti, Recent trends in the theory of boundary value problems for ordinary differential equations, Bull. Un. Mat. Ital., 22 (1967), 135-178.

9. - Equazioni differenziali ordinarie quasilineari con condizioni lineari, Ann. Math. Pura. Appl., 57 (1962), 48-62.

10. - Problems lineaires pour les equations differentielles ordinaires, Math. Nachr., 23 (1961), 161-178.

11. M. M. Day, Normed Linear Spaces, Springer-Verlag, 1962.

12. N. Dunford and J. T. Schwartz, Linear Operators, Part I, Interscience, New York, 1967.

13. S. Friedland, Non-oscillation and integral inequalities, Bull. Amer. Math. Soc., 80 (1974), 715-717.

14. —, Non-oscillation, disconjugacy and integral inequalities, Mem. Amer. Math. Soc., 7 (1976), 1-78.

15. F. R. Gantmacher, The theory of matrices, Chelsea, New York, 1966. 
16. H. Gingold, Uniqueness of nonlinear boundary value problems of second order differential systems and equations, (to appear).

17. H. Gingold and G. Gustafson, On the uniqueness of the De Vallée-Poussin problem, (to appear).

18. W. A. Goppel, Disconjugacy, Lecture notes in Mathematics, 220, Springer-Verlag, 1971.

19. Maria Grandolf, Problemi ai limiti per le equazioni differentielles multivoche, Atti. Accad. Naz. Lincei Rend, Cl Sci. Fix. Mat. Natur., 42 (1967), 355-360.

20. P. Hartman and A. Wintner, On disconjugate differential systems, Canad. J. Math., 8 (1956), $72-81$.

21. P. Hartman, Ordinary Differential Equations, Wiley, New York, 1964.

22. E. Hille, Analytic Function Theory, Ginn and Company, 1962.

23. — Lectures on Differential Equations, Addison-Wesley publishing Co., 1969.

24. D. B. Hinton, Disconjugate properties of a system of differential equations, J. Differential Equations, 2 (1966), 420-437.

25. S. Karlin, Total Positivity, Vol. I, Stanford University Press, California, 1968.

26. - Total positivity, interpolation by Splines and Green's functions of differential operators, J. Approximation Theory, 4 (1971), 91-112.

27. - Some extremal problems for eigenvalues of certain matrix and integral operators, Advances in Math., 9 (1972), 93-136.

28. W. J. Kim, Disconjugacy and disfocality of differential systems, J. Math. Anal. Appl., 26 (1969), 9-19.

29. H. B. Keller, Numerical methods for two-point boundary value problems, Blaisdell, Waltham, Massachusetts, 1968.

30. A. Lasota and Z. Opial, An application of the Kakutani-Ky fan theorem in the theory of ordinary differential equations, Bull. Acad. Polon. Sci. Ser. Sci. Math. Astronom. Phys., 13 (1965), 781-786.

31. - On the existence of solutions of linear problems for ordinary differential equations, Ibid., 14 (1966), 371-376.

32. A. Lasota and C. Olech, An optimal solution of Nicoletti's boundary value problem, Ann. Polon. Math., 18 (1966), 131-139.

33. M. Lavie, Some function-theoretic aspects of disconjugacy of linear differential systems, Trans. Amer. Math. Soc., 143 (1969), 153-171.

34. A. Ju. Levin, On certain estimates for differentiable functions, Dokl. Akad. Nauk. SSSR, 138 (1961), 37-38.

35. Some questions on the oscillation of solutions of linear differential equations, Dokl. Akad. Nauk SSSR, 148 (1963), 512-515.

36. Distribution of the zeros of solutions of a linear differential equation, Dokl. Akad. Nauk SSSR, 156 (1964), 1281-1284.

37. - Fredholm equation with a smooth kernel and boundary value problems for linear differential equations, Dokl. Akad. Nauk SSSR, 159 (1964), 13-16.

38. A. Ju. Levin, The non-oscillation of solutions of the differential equation $x^{(n)}+p_{1}(t) x^{(n-1)}+\cdots+$ $p_{n}(t) x=0^{\prime \prime}$, Uspeh. Mat. Nauk, 24 (1969), 43-96.

39. D. London and B. Schwarz, Disconjugacy of complex difierential systems and equation, Trans. Amer. Math. Soc., 135 (1969), 487-505.

40. J. L. Massera and J. J. Schaffer, Linear Differential Equations and Function Spaces, Academic Press, New York, 1966.

41. A. McNabb and Alan Shumitzky, Factorization and imbedding for general linear boundary value problems, J. Differential Equations, 14 (1973), 518-546.

42. Z. Nehari, The Schwarzian derivative and Schlicht functions, Bull. Amer. Math. Soc., 55 (1949), 545-551.

43. - Some inequalities in the Theory of functions, Trans. Amer. Math. Soc., 75 (1953), $256-286$. 
44. — Some eigenvalue estimates, J. Analyse Math., 7 (1959), 79-88.

45. - Oscillation theorems for systems of linear equations, Trans. Amer. Math. Soc., 139 (1969), 339-347.

46. Non-oscillation and disconjugacy of systems of linear differential equations, J. Math. Anal. Appl., 42 (1973), 237-254.

47. - Oscillatory properties of complex differential systems, J. Analyse Math., 26 (1973), 413-429.

48. - A minimal-length problem in $R^{n}$, J. London Math. Soc. 8 (1974), 22-28.

49. O. Nicoletti, Sulle condizionia inviziali che determinano gli integrali delle equazioni ordinaire, Atti. Accad. Sci. Torino, 33 (1897-98), 746-759.

50. W. T. Reid, Principal solutions of non-oscillatory self-adjoint linear differential equations, Pacific J. Math., 8 (1958), 147-169.

51. - Oscillation criteria for self-adjoint differential systems, Trans. Amer. Math. Soc., 101 (1961), 91-106.

52. B. Schwarz, Disconjugacy of complex differential systems, Trans. Amer. Math. Soc., 125 (1966), $482-496$.

53. - Norm conditions for disconjugacy of complex differential systems, J. Math. Anal. Appl., 28 (1969), 553-568.

54. - Mappings of domains by components of solutions of differential systems, J. Differential Equations, 10 (1971), 314-323.

55. - Curves on the unit sphere and disconjugacy of differential systems, J. Math. Anal. Appl., 39 (1972), 75-86.

56. R. L. Sternberg, Variational methods and non-oscillation theorems for systems of differential equations, Duke Math. J., 19 (1952), 311-322.

57. D. V. V. Wend, On the zeros of solutions of some linear complex differential equations, Pacific J. Math., 10 (1960), 713-722.

Received May 20, 1976 and in revised form April 6, 1977.

UNIVERSITY OF UTAH

Salt LAKe CITY, UT 84112 


\section{PACIFIC JOURNAL OF MATHEMATICS EDITORS}

ICHARD ARENS (Managing Editor)

niversity of California

os Angeles, CA 90024

\section{. A. BEAUmont}

niversity of Washington

sattle, WA 98105

. C. MOORE

niversity of California

erkeley, CA 94720
J. DUGUNDJI

Department of Mathematics

University of Southern California

Los Angeles, CA 90007

R. FINN AND J. MiLgRAM

Stanford University

Stanford, CA 94305

\section{ASSOCIATE EDITORS}
F. BECKENBACH
B. H. NEUMANN
F. WOLF
K. YosHidA

\section{SUPPORTING INSTITUTIONS}

NIVERSITY OF BRITISH COLUMBIA

UNIVERSITY OF SOUTHERN CALIFORNIA

ALIFORNIA INSTITUTE OF TECHNOLOGY STANFORD UNIVERSITY

NIVERSITY OF CALIFORNIA

ONTANA STATE UNIVERSITY

UNIVERSITY OF HAWAII

NIVERSITY OF NEVADA

UNIVERSITY OF TOKYO

EW MEXICO STATE UNIVERSITY

UNIVERSITY OF UTAH

REGON STATE UNIVERSITY

NIVERSITY OF OREGON

WASHINGTON STATE UNIVERSITY

UNIVERSITY OF WASHINGTON

SAKA UNIVERSITY

AMERICAN MATHEMATICAL SOCIETY

The Supporting Institutions listed above contribute to the cost of publication of this Journal, but they a t owners or publishers and have no responsibility for its contents or policies.

Mathematical papers intended for publication in the Pacific Journal of Mathematics should be in typ Irm or offset-reproduced (not dittoed), double spaced with large margins. Underline Greek letters in re ierman in green, and script in blue. The first paragraph or two must be capable of being used separately as 'nopsis of the entire paper. Items of the bibliography should not be cited there unless absolutely necessary, hich case they must be identified by author and Journal, rather than by item number. Manuscripts, uplicate, may be sent to any one of the four editors. Please classify according to the scheme of Math. Revieu Idex to Vol. 39. All other communications should be addressed to the managing editor, or Elaine Bart Iniversity of California, Los Angeles, California, 90024.

100 reprints are provided free for each article, only if page charges have been substantially pai dditional copies may be obtained at cost in multiples of 50 .

The Pacific Journal of Mathematics is issued monthly as of January 1966. Regular subscription rate: \$72. year (6 Vols., 12 issues). Special rate: $\$ 36.00$ a year to individual members of supporting institutions.

Subscriptions, orders for numbers issued in the last three calendar years, and changes of address should ent to Pacific Journal of Mathematics, 103 Highland Boulevard, Berkeley, California, 94708.

UBLISHED BY PACIFIC JOURNAL OF MATHEMATICS, A NON-PROFIT CORPORATION

Printed at Jerusalem Academic Press, POB 2390, Jerusalem, Israel.

Copyright (C) 1978 Pacific Journal of Mathematics

All Rights Reserved 


\section{Pacific Journal of Mathematics}

\section{Vol. 75, No. $1 \quad$ September, 1978}

Mieczyslaw Altman, General solvability theorems

Denise Amar and Eric Amar, Sur les suites d'interpolation en plusieurs variables ..........................................

Herbert Stanley Bear, Jr. and Gerald Norman Hile, Algebras which satisfy a second order linear partial differential equation ..................

Marilyn Breen, Sets in $R^{d}$ having $(d-2)$-dimensional kernels ............

Gavin Brown and William Moran, Analytic discs in the maximal ideal space

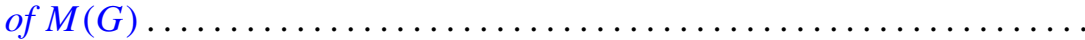

Ronald P. Brown, Quadratic forms with prescribed Stiefel-Whitney

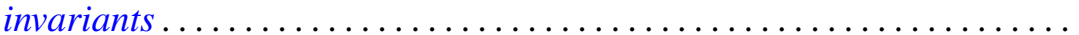

Gulbank D. Chakerian and H. Groemer, On coverings of Euclidean space by convex sets

S. Feigelstock and Z. Schlussel, Principal ideal and Noetherian groups.....

Ralph S. Freese and James Bryant Nation, Projective lattices ............

Harry Gingold, Uniqueness of linear boundary value problems for

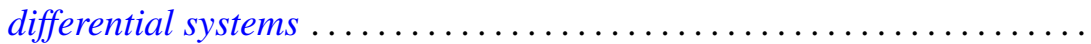

John R. Hedstrom and Evan Green Houston, Jr., Pseudo-valuation domains. . .

William Josephson, Coallocation between lattices with applications to measure extensions

M. Koskela, A characterization of non-negative matrix operators on $l^{p}$ to $l^{q}$ with $\infty>p \geq q>1$

Kurt Kreith and Charles Andrew Swanson, Conjugate points for nonlinear differential equations...........................

Shoji Kyuno, On prime gamma rings ........................ 185

Alois Andreas Lechicki, On bounded and subcontinuous multifunctions ..

Roberto Longo, A simple proof of the existence of modular automorphisms in approximately finite-dimensional von Neumann algebras ...

Kenneth Millett, Obstructions to pseudoisotopy implying isotopy for

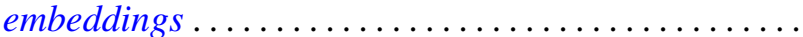

William F. Moss and John Piepenbrink, Positive solutions of elliptic equations. .

Mitsuru Nakai and Leo Sario, Duffin's function and Hadamard's

conjecture

Mohan S. Putcha, Word equations in some geometric semigroups ...

Walter Rudin, Peak-interpolation sets of class $C^{1} \ldots \ldots \ldots$

Elias Saab, On the Radon-Nikodým property in a class of locally convex

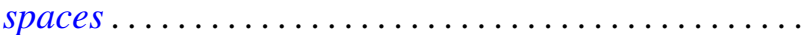

